\title{
XLVIII. On the digestion of vegetable albumen, fat and starch
}

\author{
Robert D. Thomson M.D.
}

To cite this article: Robert D. Thomson M.D. (1845) XLVIII. On the digestion of vegetable albumen, fat and starch, Philosophical Magazine Series 3, 26:173, 322-328, DOI:

10.1080/14786444508645138

To link to this article: http://dx.doi.org/10.1080/14786444508645138

Published online: 30 Apr 2009.

Submit your article to this journal $₫$

Q View related articles $\square$ 
nuation of my researches on electro-physiology, - on the subject of which I hope soon to be able to address the Royal Society, - I have not been able to continue the study of this phænomenon.

If you think it worth it, give this letter to Mr. Taylor for the Philosophical Magazine.

Pisa, March 7, 1845.

Yours faithfully,

Carlo Matteucci.

XLVIII. On the Digestion of Vegetable Albumen, Fat and Starch. By Robert D. Thomson, M.D., Lecturer on Practical Chemistry in the University of Glasgow*.

DIGESTION of Vegetable Albumen and Fat.-Nearly three years ago Dr. A. Buchanan communicated to me the fact, that he had frequently observed the serum of the blood to present a white appearance when a person happened to have been bled for some affection of such a circumscribed nature as not to interfere with the appetite or digestion; but an opportunity did not occur to me of examining this peculiar serum till the end of 1843, when Dr. Buchanan was so kind as to place some of it at my disposal. The fluid presented the appearance of thin milk, being covered, after standing, on the upper surface with a whitish scum of a denser character than that which was diffused through the fluid. When the fluid was filtered a portion of the scum remained on the filter while the liquid passed through, possessing still a milky aspect, but obviously being deprived of a considerable portion of its denser particles. Dr. Buchanan however observed that this white matter might be accumulated in larger quantities, or at least collected into smaller bulk, by saturating the serum with common salt, when the white matter speedily rose to the surface in the form of a creamy layer, which did not change its physical character even when kept for months $\dagger$. I found that the white matter, both when separated simply by the filter and also when precipitated, if we may so speak, by common salt, contained a substance which was insoluble in ether and alcohol, and that when dissolved in caustic potash and the solution was boiled with acetate of lead, a black precipitate of sulphuret of lead fell $\ddagger$. These experiments were several times repeated carefully with the same result, and were witnessed by Dr. Buchanan, so that we had no doubt in our own

* Read before the Philosophical Society of Glasgow, February 5, 1845.

+ See a paper on this sulject by Dr. Buchanan, Proceedings of the Philosophical Society of Glasgow, vol. i. p. 226.

$\ddagger$ This test for sulphur in albumen was employed in 1812 by Vauquelin. 
minds that the presence of traces of an albuminous substance in the white matter of this serum was established; and we had subsequently opportunities of obtaining this matter in larger quantities, so as to confirm the previous experiments.

These results led to a series of researches upon the effect of food on animals, and also on man; from which it was clearly demonstrated that the white colour of the serum in healthy animals is dependent on the introduction of food into the system. The detail of one or two experiments will place this conclusion in a distinct point of view. It was necessary for this purpose to observe the characters of the serum of an individual who had not tasted food for such a space of time that the effects of the previous meal should have disappeared. On the 10th of March 1844, from a stout young man, aged thirty, who had tasted no food from the preceding evening at six o'clock p.m., two ounces of blood were taken at noon, or at an interval of eighteen hours after a meal. The blood coagulated on standing in the usual manner, and the supernatant serum was found to possess a pale yellow colour, and to be perfectly clear and limpid.

After the abstraction of the blood, the individual dined upon twenty-four ounces of a pudding consisting of two parts of wheat flour and one part of suet, seasoned with salt. At three o'clock, or in about three hours after the food had been swallowed, seven ounces of blood were taken by venesection from the arm. The clot formed as usual, no appearance of a buffy coat presenting itself. The serum was whitish and opake; when heated it became more translucent, apparently from the solution of some of the solid particles diffused through it, or; as was afterwards apparent, from the liquefaction of the fatty matter diffused through the serum. The whole fluid possessed a somewhat syrupy cast of appearance, and was very heavy, its specific gravity being as high as 1029.8. Comparing this density with the average density of serum as we find it in physiological works, $1026 \frac{1}{2}$ by $\mathrm{Dr}$. Thomson, 1027 to 1029 by Müller, it must be pronounced high; but as the specific gravity of this fluid has never been properly estimated in perhaps the truly healthy condition of animals, and more especially in parallel cases with that described, no conclusion can be deduced in reference to the density of the serum as compared with the normal standard. On throwing the serum upon a filter, a portion of white matter remained attached to its interior surface, while the liquid which passed through retained still a milky aspect; but perhaps the most interesting result obtained by this experiment was, that on drying the filter and holding it between the eye and the light, it was 
found to have imbibed a considerable amount of oily matter. The white substance remaining on the filter presented characters similar to those of albuminous or fibrinous matter.

The facts detailed having shown that there was a decided distinction between the serum of a person previous to and after taking food, it became an interesting point to pursue the inquiry, and to ascertain how long the milky aspect would continue in the blood. Accordingly, at six o'clock p.m., or six hours after the meal, the individual was a third time bled to the extent of seven ounces. The serum was in this case very milky, and threw up, on standing, a considerable white scum to the surface, which could be drawn up by a sucker and examined. On throwing the serum on a filter, a small portion of white matter adhered to it, possessing albuminous characters; the serum passed through with a milky colour, but no imbibition of oil could be detected on the filter itself. A careful examination of this specimen of serum could detect not a trace of starch by means of iodine and chlorine, and this led to the investigations detailed in the subsequent parts of this paper.

The inferences deducible from this experiment appear to be, -1 st, that the serum of a healthy individual at a certain period of time, after partaking of a meal, is clear and limpid, and corresponds with the description of this fluid as we find it detailed in physiological works of authority; 2nd, that in three hours after a meal, when the food consists of vegetable albuminous matter and oil or fal, the albumen begins to make its appearance in the blood, while a still larger quantity of fat in relation to the amount of the albumen taken into the stomach exists in the blood; and 3rd, that in six hours, while the quantity of albuminous matter in the blood can be detected, the fatty matter has comparatively disappeared; a conclusion which is completely in accordance with the chenical properties of these substances, since we know that some kinds of fat liquefy nearly at the temperature of the human body, and will, of consequence, be in a condition immediately after their introduction into the stomach to enter the sanguineous circulation along with the water present in the stomach, since that fluid appears capable of permeating with great facility the coats of the intestinal canal throughout its whole length.

To determine the manner in which the food thus passes into the circulating system, is scarcely the province of the chemist. At the same time, since it appears to exist in the current of the blood without having undergone much modification of state from that which it originally possessed in the stomach, there seem no obvious arguments to present themselves against 
the conclusion that the food is directly absorbed from the alimentary canal by the blood-vessels themselves.

In confirmation of the experiment already detailed, the physiological part of which was conducted by Dr. Buchanan, whose great merits as a physiologist and original observer require no encomiums from me, various repetitions on the inferior animals were conducted by myself, which it would serve no purpose to enumerate minutely, since they all conducted to the same results.

In the majority of these cases calves were fed on gruel and milk, and after various intervals they were slaughtered. The serum on examination, when the animal was killed from three to six hours after the meal, was found to be milky, and to leave a greasy stain on filtering paper when the amount of milk or fatty matter used was considerable, while the serum taken from an animal which had been subjected to starvation for a space of time varying from twelve to twenty-four hours, presented generally a clear aspect. It has been frequently remarked that the serum of diabetic patients exhibits often a milky appearance, and the circumstance of the coexistence of disease with the white serum was considered as a proof that the disease was the cause of the colour of the liquid part of the blood; but it has been very properiy remarked by Dr. Buchanan, that the large amount of food consumed by individuals affected with this disease, affords a satisfactory mode of accounting for the presence of such profuse quantities of albuminous and fatty matter in the blood in such instances. I have recently had opportunities of studying this form of serum through the kindness of Dr. William Thomson, and I have found no reason to conclude that the blcod of diabetic patients, so far as its milkiness is concerned, is more diseased than that of healthy individuals after a full meal. It is no doubt highly probable that the blood in these instances remains for a longer time loaded with the white natter than in the healthy state of the organism, and that the appetite may, by continually urging in a new supply of food, thus produce a diminished rate of digestion or assimilation in the circulating system; but the presence of the white matter in the serum it would be erroneous to consider as a symptom of disease, at least in so far as the data entitle us to draw such an inference.

Changes produced on Starch in Digestion. - As it appeared to be a matter of importance in the investigation of the changes occurring in the stomach during digestion, in order to prevent complication of the phænomena, that the food should be as simple as possible, I have chosen for the experiments about to be detailed, cases in which animals were fed on vegetable 
food alone. In general the species of food selected was porridge, or a mixture of oatmeal and water well-boiled. In such experiments as I have seen detailed, in reference to the free acid of the stomach, there appears to have been too little attention paid to the possible results which might arise from a difference in the nature of the food. Thus the products of the digestion of starch we should naturally expect to be different from those of the digestion of animal fibrin or albumen, since we know that lactic acid can be produced by a modification of starch, although the same change does not attend the decomposition of the animal substances enumerated. For example, in the preparation of starch an acid liquor collects on the surface of the vessels in which the starch is digested, and in the formation of soroans from oat-husks and water by steeping, an acid liquor is developed, - in both instances a consequence of the production of lactic acid at the expense of starch; while in the German dish, saurkraut, the same acid is generated by an analogous action. I do not at present intend to discuss the nature of the acid which presents itself in the stomach during the digestion of animal food, but simply to detail the results of a series of experiments upon the changes produced on vegetable food and starch during digestion. I shall merely content myself with stating, that I have never found a volatile acid in the stomachs of animals which were digesting animal food alone, while in these cases I have invariably found an acid to be present which was fixed at the temperature of $212^{\circ}$, and even considerably higher.

The nature of the Acid developed during the digestion of Starch. -It does not appear an invariable rule that an acid reaction should always characterize the liquid present in the stomach during digestion, as appenrs from the following experiment. On the 1 ith of July 1844, the different stomachs of a sheep killed twenty-four hours after partaking of grass, contained between two and three pounds of finely-divided green matter exhibiting a pulpy consistence, but being entirely destitute of either an acid or alkaline reaction. The pulpy masses were tested in each of the stomachs with the same negative result, and they were afterwards mixed together, diluted with distilled water and filtered, but still without producing any effect on litmus paper. Experience teaches us, that to determine the presence of a volatile acid in the stomach, great care must be taken in reference to the mode in which the distillation is performed, since if the heat of an open fire is applied to the retort, an indication of the presence of hydrochloric acid will be found in the liquid contained in the receiver by the addition of nitrate of silver. The following experiment affords 
evidence in favour of this position, and it might be strengthened, if necessary, by the results obtained by other experimenters. 'The contents of the stomach of a dog which had been fed on porridge made of oatmeal and water, and which afforded an acid reaction with litmus paper, were mixed with distilled water and filtered. On exposing the distilled liquor to the heat of an open fire in a retort, a fluid passed over possessing an acid reaction, and becoming opalescent on the addition of a solution of nitrate of silver, and without disappearance in contact with nitric acid; . a result plainly indicative of the presence of chlorine in some form in the liquor of the receiver, but, as appeared by the subsequent inquiries, to be explained by the fact that muriate of ammonia must have been carried over by the vapour of water in consequence of the excess of heat.

To determine in a satisfactory manner therefore the presence of a volatile acid, it is necessary to distil the fluid contents of the stomach in a water-bath. With this consicleration in view the following experiment was made. June 1844, a pig was fed on potatoes and greens, and was killed in half an hour after the food was swallowed. 'The stomach was slit open, and as much of the fluid contents as possible were poured off; the solid contents were then digested in cold distilled water. Both fluids were filtered and mixed, each previous to being united heing found to exhibit an acid reaction with litmus infusion. To ascertain whether the statement made by a French physiologist (Blondlot) is correct, viz. that the acid of the stomach cannot be saturated with chalk, a quantity of pure carbonate of lime was prepared by dissolving Irish limestone in hydrochloric acid, precipitating a small portion of phosphate of lime and iron which it contains with caustic ammonia, and then throwing down the lime with carbonate of ammonia, collecting the precipitate on a filter, washing it well with distilled water, and heating it to redness in at platinum crucible. An excess of this chalk was then added to the filtered fluid of the stomach, and the mixture was allowed to remain for twentyfour hours, disturbed only by frequent agitation. It was then filtered, and found to have been completely neutralized. Having often repeated this experiment on various specimens of gastric fluid with the same effect, 1 can only account for the different result obtained by Blondlot, by supposing that he had attempted to complete his neutralization of the fluid while it was in a heated state, and that possibly, if acetic or lactic acids were present, these acids might resist neutralization at an elevated temperature, since it has been found by manufacturers of pyroligneous acid that they cannot succeed in form- 


\section{Mr. Hopkins on the Mechanism of Glacial Motion}

ing a neutral acetate of lime at a temperature approaching that of the boiling-point by chalk alone, but that they require to add milk of lime in order to overcome the acid reaction. Blondlot has deduced the inference from his experiment, that the stomach owes its acid reaction to the presence of an acid phosphate of lime; but as the experiments now detailed do not coincide with those of the French physiologist, it is sufficiently obvious that they do not support him in his conclusions.

['To be continued.]

XLIX. On the Mechanism of Glacial Motion. Fourth Letter. By W. Hopkins, Esq., M.A., F.R.S., \& c.

To the Editors of the Philosophical Magazine and Journal. Gentlemen,

SINCE I addressed my last letter to you on the motion of S glaciers, I have devised some experiments, the results of which I wish to communicate, as corroborative of the conclusions at which I have arrived by mathematical investigation.

The weight of a solid mass sufficiently small to experiment upon conveniently, is not sufficient to communicate a motion to it which, as in the case of a glacier, shall superinduce any considerable change of form and consequent fracture, the conditions under which the mass is placed being analogous to those of a glacier. When the width of a mass under such conditions is increased, the tendency of the forces arising from the weight of the mass to fracture it is also increased; and lateral obstacles, which would entirely arrest the motion of a mass of limited width, might produce scarcely a sensible effect on the central motion of a mass of large dimensions. When our object, however, is to determine the effects of an assigned motion and consequent change of form, as in the case before us, it is immaterial whether the motion be produced by the action of gravity, or any other cause. In my experiments the mass was about three feet in length and two in breadth, and two inches in depth. It consisted of fine mortar, which was allowed to assume different degrees of solidity, according to the particular object of each experiment.

The experiments were conducted as follows:-A trough of the length and width above-mentioned and five or six inches in depth, was prepared, open at both ends. Along the bottom of it was placed longitudinally a layer of straight rods parallel to each other, on which the mortar was poured, its consistency being about the same as that of mortar used in 